\title{
Primary tumor surgery improves survival in non-metastatic primary urethral carcinoma patients: a large population-based investigation
}

\author{
Jie Wu ${ }^{1,2+}$, Yu-Chen Wang ${ }^{1,2+}$, Wen-Jie Luo ${ }^{1,2}$, Bo-Dai ${ }^{1,2}$, Ding-Wei Ye ${ }^{1,2^{*}}$ and Yi-Ping Zhu ${ }^{1,2^{*}}$ (D)
}

\begin{abstract}
Background: Primary urethral carcinoma (PUC) is a rare genitourinary malignancy with a relatively poor prognosis. The aim of this study was to examine the impact of surgery on survival of patients diagnosed with PUC.

Methods: A total of 1544 PUC patients diagnosed between 2004 and 2016 were identified based on the SEER database. The Kaplan-Meier estimate and the Fine and Gray competing risks analysis were performed to assess overall survival (OS) and cancer-specific mortality (CSM). The multivariate Cox regression model and competing risks regression model were used to identify independent risk factors of OS and cancer-specific survival (CSS).

Results: The 5-yr OS was significantly better in patients who received either local therapy (39.8\%) or radical surgery (44.7\%) compared to patients receiving no surgery of the primary site $(21.5 \%)(p<0.001)$. Both local therapy and radical surgery were each independently associated with decreased CSM, with predicted 5-yr cumulative incidence of 45.4 and $43.3 \%$, respectively, compared to $64.7 \%$ for patients receiving no surgery of the primary site $(p<0.001)$. Multivariate analyses demonstrated that primary site surgery was independently associated with better OS (local therapy, $p=0.037$; radical surgery, $p<0.001)$ and decreased CSM $(p=0.003)$. Similar results were noted regardless of age, sex, T stage, N stage, and AJCC prognostic groups based on subgroup analysis. However, patients with M1 disease who underwent primary site surgery did not exhibit any survival benefit.
\end{abstract}

Conclusion: Surgery for the primary tumor conferred a survival advantage in non-metastatic PUC patients.

Keywords: Primary urethral carcinoma, Survival, SEER, Surgery

\section{Background}

Primary urethral carcinoma (PUC) is a rare genitourinary malignancy with a relatively poor prognosis [1-3]. In 2020, it was estimated that in the United States there are 3970 new diagnoses of cancer of the ureter and other urinary organs, and 1010 will die of these diseases [3].

\footnotetext{
*Correspondence: dwyeli@yahoo.com.cn; fudanzhuyiping@163.com

${ }^{\dagger}$ Jie Wu and Yu-Chen Wang contributed equally to this work.

${ }^{1}$ Department of Urology, Fudan University Shanghai Cancer Center, No. 270 Dong an Road, Shanghai 200032, People's Republic of China

Full list of author information is available at the end of the article
}

The 5-yr overall survival rate in PUC patients is reported to be $42 \%[4,5]$. Disease management of PUC is based on tumor stage, patient sex and tumor location $[2,6,7]$. Surgery, chemotherapy or radiation therapy are standard treatment options for patients diagnosed with PUC [810]. Unfortunately, owing to its rare nature, there is a lack of large-scale investigations to support the treatment strategies. The aim of this study was to examine the impact of surgery on survival of patients diagnosed with PUC using a large population-based cancer database.

(c) The Author(s). 2021 Open Access This article is licensed under a Creative Commons Attribution 4.0 International License, which permits use, sharing, adaptation, distribution and reproduction in any medium or format, as long as you give appropriate credit to the original author(s) and the source, provide a link to the Creative Commons licence, and indicate if changes were made. The images or other third party material in this article are included in the article's Creative Commons licence, unless indicated otherwise in a credit line to the material. If material is not included in the article's Creative Commons licence and your intended use is not permitted by statutory regulation or exceeds the permitted use, you will need to obtain permission directly from the copyright holder. To view a copy of this licence, visit http://creativecommons.org/licenses/by/4.0/. The Creative Commons Public Domain Dedication waiver (http://creativecommons.org/publicdomain/zero/1.0/) applies to the data made available in this article, unless otherwise stated in a credit line to the data. 


\section{Methods}

\section{Selection of patient cohort}

We searched Surveillance, Epidemiology, and End Results (SEER) public-access database covering around $27.8 \%$ of the U.S. population from 2004 to 2016 and identified patients diagnosed with PUC based on the International Classification of Diseases-O-3 (ICD-O-3) codes C68.0. Only patients who met the following criteria were included: (1) urethra was the primary site; (2) survival time was $\geq 1$ month; and (3) adequate tumor data were available. Data were extracted from the SEER database using SEER*Stat Software (version 8.3.6).

\section{Data collection and variable definition}

Parameters of interest included race, sex, age at diagnosis, the American Joint Commission on Cancer (AJCC) TNM Staging system, histology, tumor size, and grade. Therapy and follow-up information including type of surgical procedure, radiation, chemotherapy, survival months, and vital status were also collected. Surgical codes 30 (Simple/partial surgical removal of primary site), 40 (Total surgical removal of primary site; enucleation), 50 (Surgery stated to be "debulking"), and 60 (Radical surgery) for PUC were merged and collectively defined as "radical surgery". Transurethral resection and other local tumor destruction or excision procedures (Surgical codes 10 and 20) were merged and collectively defined as "local therapy". Surgical codes 00 (no surgery of primary site or autopsy only) was defined as "No surgery of primary site". The overall survival (OS) months for PUC were defined as the time from diagnosis to any cause of death or last follow-up, with patients still alive censored at the last follow-up. For cancer-specific mortality (CSM), deaths not due to PUC were considered as competing risks.

\section{Statistical analysis}

Pearson's chi-square was applied to compare the distribution of categorical data. Kaplan-Meier survival curves and log-rank tests were utilized to perform survival analysis. The Fine and Gray competing risk analysis was used to evaluate CSM $[11,12]$. Multivariate Cox regression and competing risk regression analysis were utilized to identify independent risk factors to predict OS and cancer-specific survival (CSS) of PUC patients. All tests were two sided with a statistical significance set at $p<$ 0.05 . Statistical analyses were performed using $R$ version 3.5.2 (the $\mathrm{R}$ foundation for Statistical Computing, Vienna, Austria).

\section{Results}

Demographic and clinical characteristics of PUC patients A total of 1544 PUC patients were identified. The demographic and clinical characteristics of the patient cohort are listed in Table 1. The majority of PUC patients were white $(1164,75.4 \%)$, male $(971,62.9 \%)$, with stage I (432, $28.2 \%)$ or IV (392, 25.6\%) TNM stage, and III/ IV grade (833, 54.0\%). Among 1544 PUC patients, 642 patients have precise tumor sizes, and the median size was 38.27 $\mathrm{mm}$. Median age at diagnosis was 69.54 years. The pathological types comprised squamous cell carcinoma (437, 28.3\%), transitional cell carcinoma (660, 42.7\%), adenocarcinoma $(252,16.3 \%)$, and other pathological types $(195,12.6 \%)$. With regard to therapy, most patients underwent a surgical procedure $(1114,72.2 \%)$, and did not receive radiation $(1141,73.9 \%)$ or chemotherapy (1067, 69.1\%).

\section{Survival analyses in the overall patient cohort stratified by surgical procedure}

Among the 1544 PUC patients, 403 (26.1\%) did not undergo any surgery to the primary tumor, 532 (34.5\%) received local therapy (transurethral or transvaginal resection), and 582 (37.7\%) underwent radical surgery (urethrectomy). Patient characteristics stratified by surgical procedure are also presented in Table 1. The 5-yr OS was significantly better in patients undergoing either local therapy (39.8\%; 95\% CI: 35.3-44.7) or radical surgery (44.7\%; 95\% CI: 40.1-49.7) compared to patients receiving no surgery of the primary site $(21.5 \%$; $95 \% \mathrm{CI}$ : $17.4-26.7)(p<0.001)$ (Fig. 1 and Table 4$)$. In addition, undergoing local therapy or radical surgery was each independently associated with decreased CSM, with predicted 5-yr cumulative incidence of 45.4 and $43.3 \%$, respectively, compared to $64.7 \%$ for patients receiving no surgery of the primary site $(p<0.001)$ (Fig. 1 and Table $4)$.

\section{Multivariate cox regression analysis and multivariable competing risks regression analysis}

Based on the univariate and multivariate Cox regression model; older age, advanced $\mathrm{T}$ stage, lymph node involvement, metastatic disease, and larger tumor size were identified as independent risk factors associated with poorer OS (Table 2). Using a multivariable competing risks regression model, the factors independently associated with increased CSM for PUC patients were identified, included; older age, metastatic disease, advanced AJCC stage groups, and larger tumor size (Table 3). Notably, surgery of the primary site was independently associated with better OS (local therapy, $p=0.037$; radical surgery, $p<0.001)$ and decreased CSM $(p=0.003)$.

\section{Subgroup survival analyses based on the risk factors}

Subgroup analyses were performed to further evaluate survival benefit of surgery for PUC patients among groups based on age ( $<70$ vs $\geq 70$ years), tumor size (< 30 vs $\geq 30 \mathrm{~mm}$ ) or sex. Patients who underwent surgery 
Table 1 Demographic and clinical characteristics of PUC patients

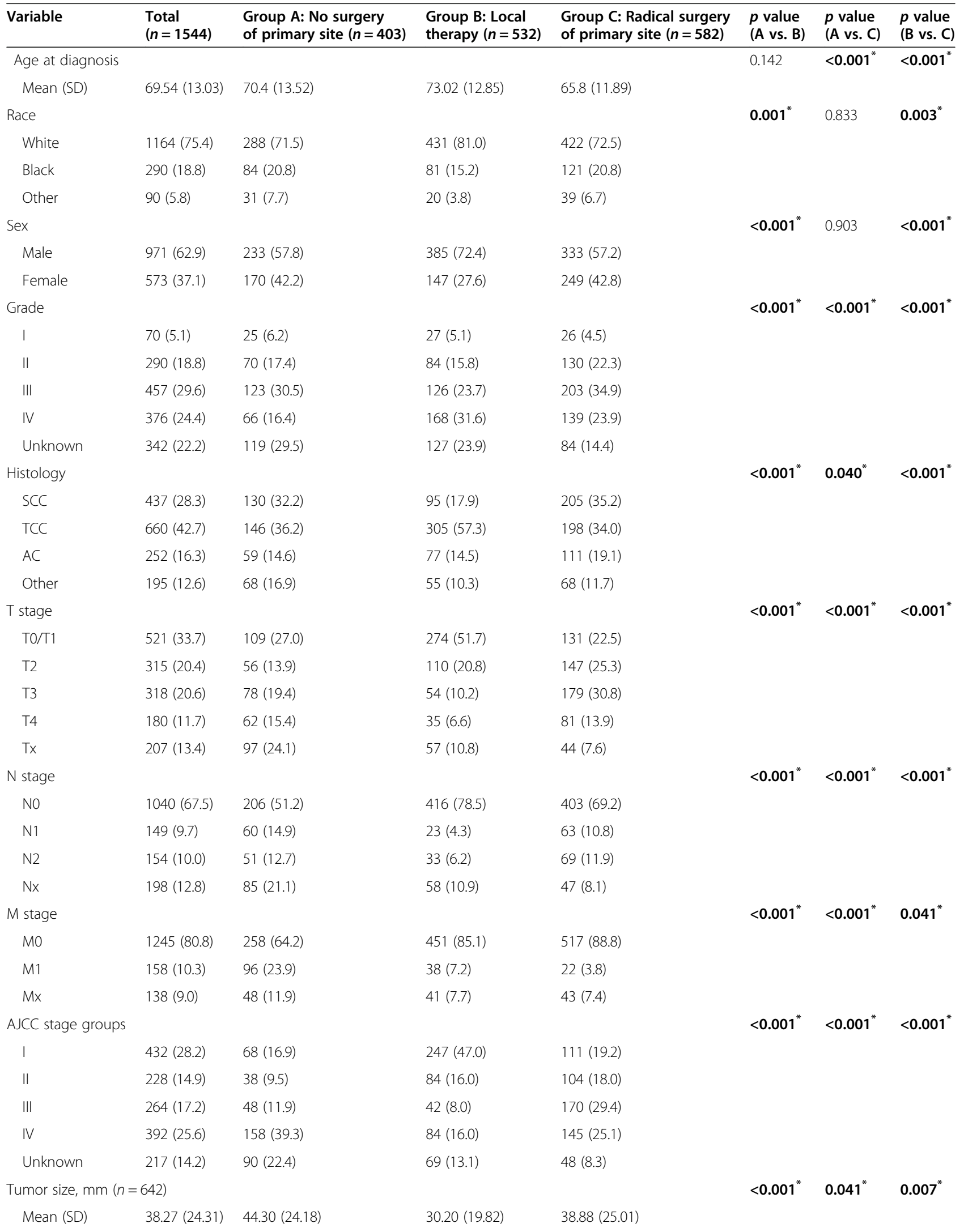


Table 1 Demographic and clinical characteristics of PUC patients (Continued)

\begin{tabular}{|c|c|c|c|c|c|c|c|}
\hline Variable & $\begin{array}{l}\text { Total } \\
(n=1544)\end{array}$ & $\begin{array}{l}\text { Group A: No surgery } \\
\text { of primary site }(n=403)\end{array}$ & $\begin{array}{l}\text { Group B: Local } \\
\text { therapy }(n=532)\end{array}$ & $\begin{array}{l}\text { Group C: Radical surgery } \\
\text { of primary site }(n=582)\end{array}$ & $\begin{array}{l}p \text { value } \\
\text { (A vs. B) }\end{array}$ & $\begin{array}{l}p \text { value } \\
\text { (A vs. C) }\end{array}$ & $\begin{array}{l}p \text { value } \\
\text { (B vs. C) }\end{array}$ \\
\hline Radiation & & & & & $<0.001^{*}$ & $<0.001^{*}$ & 0.641 \\
\hline Yes & $403(26.1)$ & $146(36.2)$ & $113(21.2)$ & $116(19.9)$ & & & \\
\hline No/Unknown & $1141(73.9)$ & $257(63.8)$ & $419(78.8)$ & $466(80.1)$ & & & \\
\hline Chemotherapy & & & & & $<0.001^{*}$ & $0.006^{*}$ & $0.011^{*}$ \\
\hline Yes & 477 (30.9) & $161(40.0)$ & $129(24.2)$ & $182(31.3)$ & & & \\
\hline No/Unknown & $1067(69.1)$ & $242(60.0)$ & 403 (75.8) & $400(68.7)$ & & & \\
\hline
\end{tabular}

PUC Primary urethral carcinoma, AJCC American Joint Committee on Cancer

of the primary site showed significant survival advantage in both age subgroups (Supplementary Figure 1 and Table 4). The benefit of surgery was more marked in patients aged $<70$ years, with median survival months of 105 and 84 for local therapy and radical surgery, respectively, compared to 21 for patients receiving no surgery of the primary site. Patients who underwent radical surgery exhibited higher OS and decreased CSM regardless of tumor size (Supplementary Figure 2 and Table 4). In contrast there were no significant differences in survival between patients who received local therapy or no surgery of the primary site in either tumor size subgroup. Subset analyses based on sex also revealed that surgery of the primary site brought significant survival benefit regardless of sex (Supplementary Figure 3 and Table 4). To determine whether higher cancer stage affected survival among surgery groups, subset analyses were also performed based on AJCC stage groups. Patients who underwent radical surgery exhibited higher OS and decreased CSM regardless of stage groups (Supplementary Figure 4 and Table 4). Local therapy did not result in significantly greater OS compared to no surgery of the primary site in the I/II stage $(p=0.392)$ or the III/IV stage group $(p=0.053)$, but did result in longer median survival (52 months) compared to no surgery (30 months) in the I/II stage group. Patients who underwent surgery of the primary site showed significant survival advantage in M0 disease, but did not exhibit any benefit in M1 disease (Supplementary Figure 5 and Table 4).

\section{Cox's and competing risks' proportional hazard analyses}

Finally, Cox's and competing risks' proportional hazard analyses were performed to assess the prognostic value of surgery in PUC patients (Fig. 2). Surgery of the primary site independently predicted statistically significantly higher OS and CSS in both age group ( $<70$ years, OS: $p<0.001$, CSS: $p<0.001$; $\geq 70$ years, OS: $p<0.001$, CSS: $p<0.001$ ), both AJCC T stages (T0/T1/T2, OS: $p<$ 0.001 , CSS: $p<0.001$; T3/T4, OS: $p<0.001$, CSS: $p<$ 0.001 ), both AJCC N stages (N0, OS: $p<0.001$, CSS: $p<$ 0.001; N1/N2, OS: $p<0.001$, CSS: $p<0.001)$, both AJCC stage groups (I/ II, OS: $p=0.013$, CSS: $p<0.001$; III $/ \mathrm{IV}$,
OS: $p<0.001$, CSS: $p=0.034$ ), both sexes (male, OS: $p<$ 0.001, CSS: $p<0.001$; female, OS: $p<0.001$, CSS: $p<$ 0.001 ), the larger tumor size group (OS: $p<0.001$, CSS: $p<0.001$ ), and the M0 group (OS: $p<0.001$, CSS: $p<$ 0.001 ), but surgery of the primary site was not an independent risk factor in the M1 group (OS: $p=0.374$, CSS: $p=0.640$ ) or the other histology group (OS: $p=0.074$, CSS: $p=0.067$ ). Notably, surgery of the primary site was an independent risk factor in the smaller tumor size group based only on the competing risks' proportional hazard analyses $(p=0.002)$.

\section{Discussion}

PUC is an aggressive and rare carcinoma, comprising < $1 \%$ of all genitourinary malignancies $[7,13]$. The disease management of PUC requires multimodal therapy to improve functional outcome and quality of life. According to the National Comprehensive Cancer Network (NCCN) and European Association of Urology (EAU) guidelines, partial urethrectomy or urethra-sparing surgery is a valid treatment option for localized distal tumors (I/II stage), and Ta-Tis-T1 PUC can also be treated with a repeat transurethral or transvaginal resection. For patients with locally advanced disease (III/IV stage), multimodal treatment strategies are needed to optimize local control and prognosis. Chemotherapy followed by surgery or radiation therapy and concurrent chemoradiation with or without surgery have been shown to lead to an improvement in survival $[2,7,14$, $15]$. However, given the rarity of PUC, there are few prospective multi-institutional studies to compare the effectiveness of various multimodal therapies, and the role of surgery in the management of PUC remains contentious.

To our knowledge, this is the first large populationbased study to investigate the benefit of surgery for PUC patients. Our results demonstrated that PUC patients who underwent radical surgery or local therapy had a higher 5-yr OS and decreased CSM compared with patients who did not receive surgery of the primary site. Subgroup analysis based on TNM stage also demonstrated that survival of PUC patients who underwent 


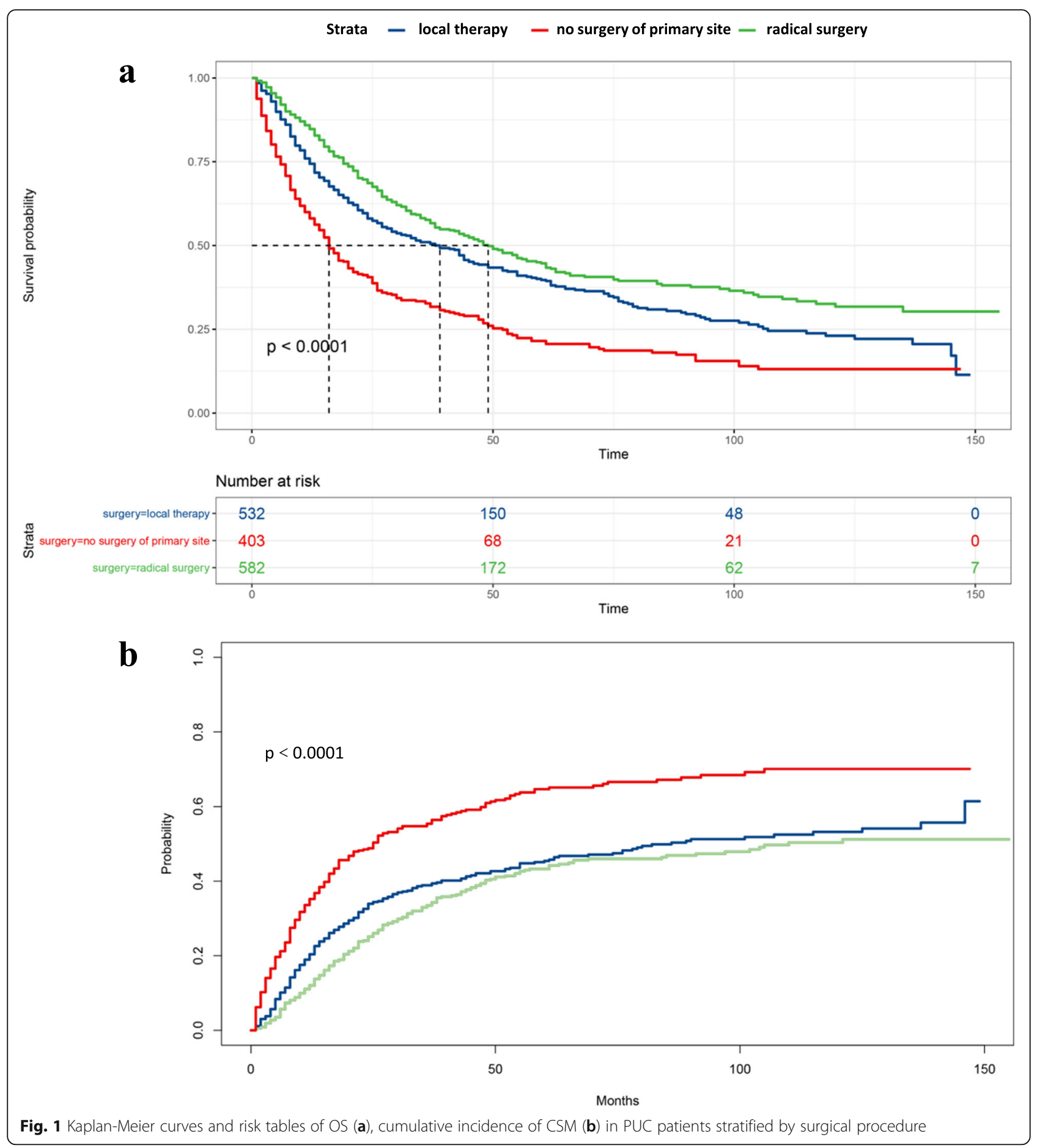

surgery of the primary site was improved regardless of $\mathrm{T}$ stage, N stage, or AJCC prognostic group. In terms of M stage, PUC patients with metastatic disease were less likely to benefit from surgery. Notably, PUC patients with early TNM stage (I/II) who received radical surgery showed a more marked survival benefit, indicating that these patients were optimal candidates for urethrectomy.
We noted that factors independently associated with poor OS and increased CSM in PUC patients other than advanced TNM stage included age $\geq 70$ years and tumor size $\geq 30 \mathrm{~mm}$. Subset analyses revealed that patients $<70$ years and tumor size $\geq 30 \mathrm{~mm}$ had a notably better survival benefit from surgery. Nevertheless, surgery of the primary site independently predicted significantly better 
Table 2 Univariate and multivariate Cox regression analysis for predicting OS of PUC

\begin{tabular}{|c|c|c|c|c|c|c|}
\hline & \multicolumn{3}{|c|}{ Univariate analysis } & \multicolumn{3}{|c|}{ Multivariate analysis } \\
\hline & $\overline{\mathrm{HR}}$ & $95 \% \mathrm{Cl}$ & $\overline{p \text { value }}$ & $\overline{\mathrm{HR}}$ & $95 \% \mathrm{Cl}$ & $p$ value \\
\hline \multicolumn{7}{|l|}{ Age } \\
\hline$<70$ & Ref & & & Ref & & \\
\hline$\geq 70$ & 1.906 & $1.667-2.179$ & $<0.001^{*}$ & 1.948 & $1.693-2.242$ & $<0.001^{*}$ \\
\hline \multicolumn{7}{|l|}{ Sex } \\
\hline Male & Ref & & & & & \\
\hline Female & 0.919 & $0.803-1.051$ & 0.281 & & & \\
\hline \multicolumn{7}{|l|}{ Race } \\
\hline White & Ref & & & & & \\
\hline Black & 1.102 & $0.937-1.297$ & 0.241 & & & \\
\hline Other & 1.050 & $0.786-1.404$ & 0.741 & & & \\
\hline \multicolumn{7}{|l|}{ Grade } \\
\hline I & Ref & & & Ref & & \\
\hline$\|$ & 1.192 & $0.847-1.678$ & 0.313 & 1.207 & $0.893-1.632$ & 0.220 \\
\hline III & 1.407 & $1.014-1.953$ & $0.041^{*}$ & 1.156 & $0.862-1.550$ & 0.334 \\
\hline IV & 1.342 & $0.961-1.874$ & 0.085 & 1.214 & $0.895-1.646$ & 0.213 \\
\hline \multicolumn{7}{|l|}{ Histology } \\
\hline SCC & Ref & & & & & \\
\hline TCC & 1.122 & $0.957-1.314$ & 0.155 & 0.899 & $0.749-1.079$ & 0.251 \\
\hline$A C$ & 0.906 & $0.737-1.114$ & 0.301 & 0.765 & $0.619-0.946$ & $0.014^{*}$ \\
\hline Other & 1.289 & $1.037-1.601$ & $0.022^{*}$ & 0.898 & $0.717-1.126$ & 0.352 \\
\hline \multicolumn{7}{|l|}{ T stage } \\
\hline T0/T1 & Ref & & & Ref & & \\
\hline $\mathrm{T} 2$ & 1.105 & $0.913-1.337$ & 0.307 & 1.125 & $0.773-1.636$ & 0.539 \\
\hline $\mathrm{T} 3$ & 1.577 & $1.313-1.894$ & $<0.001^{*}$ & 1.444 & $1.059-1.969$ & $0.020^{*}$ \\
\hline $\mathrm{T} 4$ & 2.144 & $1.738-2.646$ & $<0.001^{*}$ & 1.788 & $1.273-2.512$ & $<0.001$ \\
\hline \multicolumn{7}{|l|}{$\mathrm{N}$ stage } \\
\hline No & Ref & & & Ref & & \\
\hline N1 & 1.530 & $1.239-1.891$ & $<0.001^{*}$ & 1.057 & $0.837-1.333$ & 0.643 \\
\hline $\mathrm{N} 2$ & 1.810 & $1.464-2.238$ & $<0.001^{*}$ & 1.413 & $1.073-1.862$ & $0.014^{*}$ \\
\hline \multicolumn{7}{|l|}{ M stage of $B C$} \\
\hline MO & Ref & & & Ref & & \\
\hline M1 & 4.646 & $3.845-5.615$ & $<0.001^{*}$ & 3.080 & $2.377-3.990$ & $<0.001^{*}$ \\
\hline \multicolumn{7}{|c|}{ AJCC stage groups } \\
\hline । & Ref & & & Ref & & \\
\hline$\|$ & 1.075 & $0.859-1.345$ & 0.528 & 1.015 & $0.661-1.558$ & 0.947 \\
\hline III & 1.271 & $1.030-1.570$ & $0.026^{*}$ & 1.037 & $0.722-1.490$ & 0.843 \\
\hline IV & 2.685 & $2.247-3.209$ & $<0.001^{*}$ & 0.973 & $0.658-1.438$ & 0.891 \\
\hline \multicolumn{7}{|l|}{ Tumor size, mm } \\
\hline$<30$ & Ref & & & Ref & & \\
\hline$\geq 30$ & 1.662 & $1.307-2.115$ & $<0.001^{*}$ & 1.369 & $1.176-1.594$ & $<0.001^{*}$ \\
\hline \multicolumn{7}{|l|}{ Surgery } \\
\hline No surgery & Ref & & & Ref & & \\
\hline Local therapy & 0.599 & $0.511-0.701$ & $<0.001^{*}$ & 0.832 & $0.700-0.989$ & $0.037^{*}$ \\
\hline
\end{tabular}


Table 2 Univariate and multivariate Cox regression analysis for predicting OS of PUC (Continued)

\begin{tabular}{|c|c|c|c|c|c|c|}
\hline & \multicolumn{3}{|c|}{ Univariate analysis } & \multicolumn{3}{|c|}{ Multivariate analysis } \\
\hline & HR & $95 \% \mathrm{Cl}$ & $p$ value & HR & $95 \% \mathrm{Cl}$ & $p$ value \\
\hline Radical therapy & 0.458 & $0.389-0.539$ & $<0.001^{*}$ & 0.626 & $0.524-0.746$ & $<0.001^{*}$ \\
\hline \multicolumn{7}{|l|}{ Radiation } \\
\hline No/Unknown & Ref & & & & & \\
\hline Yes & 1.003 & $0.866-1.162$ & 0.966 & & & \\
\hline \multicolumn{7}{|l|}{ Chemotherapy } \\
\hline No/Unknown & Ref & & & & & \\
\hline Yes & 0.984 & $0.855-1.134$ & 0.826 & & & \\
\hline
\end{tabular}

OS Overall survival, PUC Primary urethral carcinoma, HR Hazard ratio, Cl Confidence interval, Ref Reference, AJCC American Joint Committee on Cancer Grade: Grade I (Well differentiated); Grade II (Moderately differentiated); Grade III (Poorly differentiated); Grade IV (Undifferentiated)

Table 3 Multivariable competing risks regression analysis for predicting CSS of PUC

\begin{tabular}{|c|c|c|c|}
\hline & \multicolumn{3}{|c|}{ Multivariable competing risks regression analysis } \\
\hline & SHR & $95 \% \mathrm{Cl}$ & $p$ value \\
\hline \multicolumn{4}{|l|}{ Age } \\
\hline$<70$ & Ref & & \\
\hline$\geq 70$ & 1.456 & $1.249-1.698$ & $<0.001^{*}$ \\
\hline \multicolumn{4}{|l|}{ Grade } \\
\hline |/ $\mid$ & Ref & & \\
\hline III /IV & 1.116 & $0.939-1.327$ & 0.213 \\
\hline \multicolumn{4}{|l|}{ Histology } \\
\hline SCC/TCC/AC & Ref & & \\
\hline Other & 1.008 & $0.795-1.276$ & 0.950 \\
\hline \multicolumn{4}{|l|}{ T stage } \\
\hline $\mathrm{TO} / \mathrm{T} 1 / \mathrm{T} 2$ & Ref & & \\
\hline $\mathrm{T} 3 / \mathrm{T} 4$ & 1.076 & $0.811-1.427$ & 0.613 \\
\hline \multicolumn{4}{|l|}{ N stage } \\
\hline No & Ref & & \\
\hline N1/N2 & 1.264 & $0.997-1.603$ & 0.053 \\
\hline \multicolumn{4}{|l|}{ M stage } \\
\hline MO & Ref & & \\
\hline M1 & 2.556 & $1.986-3.290$ & $<0.001^{*}$ \\
\hline \multicolumn{4}{|l|}{ AJCC stage groups } \\
\hline |/ II & Ref & & \\
\hline III /IV & 1.469 & $1.059-2.039$ & $0.021^{*}$ \\
\hline \multicolumn{4}{|l|}{ Tumor size, mm } \\
\hline$<30$ & Ref & & \\
\hline$\geq 30$ & 1.311 & $1.095-1.568$ & $0.003^{*}$ \\
\hline \multicolumn{4}{|l|}{ Surgery } \\
\hline No surgery & Ref & & \\
\hline Local/Radical therapy & 0.760 & $0.636-0.908$ & $0.003^{*}$ \\
\hline
\end{tabular}

CSS Cancer-specific survival, PUC Primary urethral carcinoma, SHR Subdistribution hazard ratio $\mathrm{Cl}$ Confidence interval, Ref Reference, AJCC American Joint Committee on Cancer 
Table 4 Subset analyses of survival of PUC patients based on age at diagnosis, AJCC 8th M stage, AJCC 8th stage groups and tumor size and sex

\begin{tabular}{|c|c|c|c|c|c|c|c|c|}
\hline \multirow[t]{2}{*}{ Overall cohort } & \multirow[t]{2}{*}{$n$} & \multirow[t]{2}{*}{ Median Survival (month) } & \multirow[t]{2}{*}{$3-y r$ OS, \% } & \multirow[t]{2}{*}{$5-y r$ OS, \% } & \multirow[t]{2}{*}{$p$ value } & \multicolumn{3}{|c|}{ CSM, \% } \\
\hline & & & & & & $1-y r$ & $3-y r$ & $5-y r$ \\
\hline No surgery of primary site & 403 & $16(14-20)$ & $32.7(28.2-38.0)$ & $21.5(17.4-26.7)$ & $<0.001^{* a}$ & 35.2 & 55.4 & 64.7 \\
\hline Local therapy & 532 & $39(29-45)$ & $51.0(46.7-55.7)$ & $39.8(35.3-44.7)$ & $0.001^{*} \mathrm{~b}$ & 20.4 & 38.9 & 45.4 \\
\hline Radical surgery of primary site & 582 & $49(42-60)$ & $57.7(53.4-62.3)$ & $44.7(40.1-49.7)$ & $<0.001^{*} \mathrm{c}$ & 12.0 & 33.5 & 43.3 \\
\hline \multirow[t]{2}{*}{ Age, yr } & \multirow[t]{2}{*}{$n$} & \multirow[t]{2}{*}{ Median Survival (month) } & \multirow[t]{2}{*}{$3-y r$ OS, \% } & \multirow[t]{2}{*}{$5-\mathrm{yr}$ OS, \% } & \multirow[t]{2}{*}{$p$ value } & \multicolumn{3}{|c|}{ CSM, \% } \\
\hline & & & & & & $1-y r$ & $3-y r$ & $5-y r$ \\
\hline \multicolumn{9}{|l|}{$<70$} \\
\hline No surgery of primary site & 178 & $21(16-36)$ & $40.9(33.9-49.4)$ & $24.7(18.3-33.4)$ & $<0.001^{*}$ & 27.6 & 53.6 & 68.9 \\
\hline Local therapy & 184 & $105(74-N A)$ & $69.3(62.5-76.7)$ & $58.9(51.4-67.4)$ & 0.465 & 11.8 & 26.4 & 32.8 \\
\hline Radical surgery of primary site & 354 & $84(56-117)$ & $65.1(59.9-70.8)$ & $53.8(48.1-60.3)$ & $<0.001^{*}$ & 10.5 & 29.2 & 38.0 \\
\hline \multicolumn{9}{|l|}{$\geq 70$} \\
\hline No surgery of primary site & 225 & $12(9-17)$ & $26.4(20.9-33.3)$ & $19.2(14.3-26.0)$ & $<0.001^{*}$ & 41.2 & 56.8 & 61.1 \\
\hline Local therapy & 348 & $25(21-30)$ & $41.6(36.4-47.4)$ & $30.0(25.0-35.9)$ & 0.059 & 24.8 & 45.3 & 51.9 \\
\hline Radical surgery of primary site & 228 & $32(27-38)$ & $45.8(39.1-53.7)$ & $30.1(23.7-38.2)$ & $<0.001^{*}$ & 14.5 & 40.4 & 51.7 \\
\hline \multirow[t]{2}{*}{ AJCC 8th M stage } & \multirow[t]{2}{*}{$n$} & \multirow[t]{2}{*}{ Median Survival (month) } & \multirow[t]{2}{*}{$3-y r$ OS, \% } & \multirow[t]{2}{*}{$5-y r$ OS, \% } & \multirow[t]{2}{*}{$p$ value } & \multicolumn{3}{|c|}{ CSM, \% } \\
\hline & & & & & & $1-y r$ & $3-y r$ & $5-y r$ \\
\hline MO & & & & & & & & \\
\hline No surgery of primary site & 258 & $25(20-31)$ & $42.2(36.2-49.1)$ & $29.2(23.5-36.2)$ & $0.001^{*}$ & 23.8 & 46.2 & 57.4 \\
\hline Local therapy & 451 & $44(37-55)$ & $54.8(50.2-59.9)$ & $42.7(37.9-48.2)$ & $0.002^{*}$ & 15.3 & 34.0 & 40.9 \\
\hline Radical surgery of primary site & 517 & $54(47-63)$ & $61.0(56.5-65.8)$ & $47.0(42.2-52.4)$ & $<0.001^{*}$ & 9.0 & 30.0 & 40.3 \\
\hline M1 & & & & & & & & \\
\hline No surgery of primary site & 96 & $7(5-9)$ & NA & NA & 0.539 & 66.3 & NA & NA \\
\hline Local therapy & 38 & $7(5-11)$ & NA & NA & $0.007^{*}$ & 71.1 & NA & NA \\
\hline Radical surgery of primary site & 22 & $10(6-31)$ & NA & NA & $0.040^{*}$ & 52.2 & NA & NA \\
\hline AJCC stage groups & $\mathrm{n}$ & Median Survival (month) & $3-y r$ OS, \% & $5-y r$ OS, \% & $p$ value & CSM, & & \\
\hline & & & & & & $1-y r$ & $3-y r$ & $5-y r$ \\
\hline$|/| \mid$ & & & & & & & & \\
\hline No surgery of primary site & 106 & $30(21-61)$ & $48.4(39.5-59.3)$ & $39.6(30.8-51.0)$ & 0.392 & 19.4 & 35.9 & 42.0 \\
\hline Local therapy & 331 & $52(43-65)$ & $57.9(52.6-63.8)$ & $46.1(40.5-52.5)$ & $<0.001^{*}$ & 13.3 & 29.5 & 35.6 \\
\hline Radical surgery of primary site & 215 & $102(76-N A)$ & $74.8(68.7-81.6)$ & $62.5(55.2-70.6)$ & $<0.001^{*}$ & 2.0 & 14.7 & 24.7 \\
\hline III /IV & & & & & & & & \\
\hline No surgery of primary site & 206 & $14(12-16)$ & $25.2(19.5-36.7)$ & $12.9(8.3-19.9)$ & 0.053 & 44.6 & 68.1 & 79.7 \\
\hline Local therapy & 126 & $16(11-23)$ & $31.8(24.0-42.0)$ & $21.7(17.4-32.0)$ & $<0.001^{*}$ & 38.6 & 62.1 & 69.7 \\
\hline Radical surgery of primary site & 315 & $35(28-45)$ & $48.3(42.5-54.8)$ & $34.3(28.5-41.4)$ & $<0.001^{*}$ & 16.7 & 43.6 & 53.6 \\
\hline Tumor size, mm & $n$ & Median Survival (month) & $3-\mathrm{yr}$ OS, \% & $5-\mathrm{yr}$ OS, \% & $p$ value & CSM, & & \\
\hline & & & & & & $1-y r$ & $3-y r$ & $5-y r$ \\
\hline$<30$ & & & & & & & & \\
\hline No surgery of primary site & 32 & $44(28-N A)$ & $60.4(44.2-82.4)$ & $34.5(16.8-70.6)$ & 0.340 & 19.5 & 35.5 & 61.4 \\
\hline Local therapy & 60 & $69(35-N A)$ & $61.9(49.6-77.2)$ & $52.7(40.2-69.2)$ & 0.086 & 9.1 & 26.2 & 30.8 \\
\hline Radical surgery of primary site & 136 & NA & $71.3(63.2-80.4)$ & $58.8(49.4-70.1)$ & $0.032^{*}$ & 3.9 & 19.3 & 30.1 \\
\hline$\geq 30$ & & & & & & & & \\
\hline No surgery of primary site & 92 & $16(12-25)$ & $29.7(21.1-41.8)$ & $18.7(11.4-30.5)$ & 0.366 & 39.3 & 62.7 & 72.1 \\
\hline Local therapy & 62 & $19(12-38)$ & $36.4(25.4-52.3)$ & $21.9(12.6-37.9)$ & $0.005^{*}$ & 30.8 & 53.2 & 60.5 \\
\hline
\end{tabular}


Table 4 Subset analyses of survival of PUC patients based on age at diagnosis, AJCC 8th M stage, AJCC 8th stage groups and tumor size and sex (Continued)

\begin{tabular}{|c|c|c|c|c|c|c|c|c|}
\hline Radical surgery of primary site & 252 & $37(30-48)$ & $50.0(43.7-57.3)$ & $37.7(31.2-45.4)$ & $<0.001^{*}$ & 16.8 & 42.5 & 52.2 \\
\hline \multirow[t]{2}{*}{ Sex } & \multirow[t]{2}{*}{$n$} & \multirow[t]{2}{*}{ Median Survival (month) } & \multirow[t]{2}{*}{$3-y r$ OS, \% } & \multirow[t]{2}{*}{$5-y r$ OS, \% } & \multirow[t]{2}{*}{$p$ value } & \multicolumn{3}{|c|}{$\mathrm{CSM}, \%$} \\
\hline & & & & & & $1-y r$ & $3-y r$ & $5-y r$ \\
\hline \multicolumn{9}{|l|}{ Male } \\
\hline No surgery of primary site & 233 & $16(12-20)$ & $32.7(26.8-39.8)$ & $23.2(17.8-30.2)$ & $<0.001^{*}$ & 39.3 & 56.6 & 63.5 \\
\hline Local therapy & 385 & $38(28-46)$ & $51.1(46.1-56.6)$ & $39.1(34.0-45.0)$ & $0.025^{*}$ & 21.4 & 38.8 & 45.0 \\
\hline Radical surgery of primary site & 333 & $46(37-60)$ & $55.8(50.1-62.1)$ & $42.5(36.5-49.5)$ & $<0.001^{*}$ & 13.1 & 33.4 & 42.8 \\
\hline \multicolumn{9}{|l|}{ Female } \\
\hline No surgery of primary site & 170 & $16(15-25)$ & $32.8(26.1-41.2)$ & $19.0(13.2-27.5)$ & $<0.001^{*}$ & 29.7 & 53.8 & 66.7 \\
\hline Local therapy & 147 & $39(25-76)$ & $50.7(42.5-60.5)$ & $41.3(33.1-51.6)$ & $0.045^{*}$ & 17.6 & 39.4 & 46.8 \\
\hline Radical surgery of primary site & 249 & $52(44-84)$ & $60.1(53.9-67.1)$ & $47.5(40.9-55.1)$ & $<0.001^{*}$ & 10.6 & 33.6 & 43.7 \\
\hline
\end{tabular}

PUC Primary urethral carcinoma, AJCC American Joint Committee on Cancer, OS Overall survival, CSM Cancer-specific mortality

a comparing survival of patients with no surgery of primary site to patients with local therapy

b comparing survival of patients with local therapy to patients with radical surgery

c comparing survival of patients with no surgery of primary site to patients with radical surgery

prognosis in both age subgroups. Several studies have demonstrated anatomic differences between male and female PUC patients that contribute to variations in clinicopathological characteristics, including tumor location and histology $[10,16,17]$. In contrast we did not observe any difference in survival between males and females, and surgery of the primary site independently predicted statistically significantly higher OS and decreased CSM in both male and female PUC patients. SCC, TCC and AC together comprised approximately $90 \%$ of the histological types of PUC, and previous studies have demonstrated poorer survival in rare PUC pathological types

\begin{tabular}{|c|c|c|c|c|c|c|c|c|c|}
\hline & Total & Surgery & No surgery & & OS & $p$ value & & cSS & $p$ value \\
\hline Overall & 1544 & $1114(72.2)$ & $403(26.1)$ & - & $0.523(0.455-0.601)$ & $<0.001^{*}$ & - & $0.531(0.453-0.622)$ & $<0.001^{\star}$ \\
\hline \multicolumn{10}{|l|}{ Age } \\
\hline$<70$ & 731 & $538(73.6)$ & $178(24.4)$ & $\mapsto$ & $0.419(0.336-0.523)$ & $<0.001^{*}$ & $\mapsto$ & $0.398(0.313-0.504)$ & $<0.001^{*}$ \\
\hline$\geq 70$ & 813 & $576(70.8)$ & $225(27.7)$ & $\longmapsto$ & $0.621(0.518-0.743)$ & $<0.001^{*}$ & $\longmapsto$ & $0.661(0.532-0.821)$ & $<0.001^{*}$ \\
\hline \multicolumn{10}{|l|}{ T stage } \\
\hline То/Т1/T2 & 836 & $662(79.2)$ & $165(19.7)$ & $\longmapsto$ & $0.603(0.486-0.749)$ & $<0.001^{*}$ & $\longmapsto$ & $0.585(0.452-0.758)$ & $<0.001^{*}$ \\
\hline $\mathrm{T} 3 / \mathrm{T} 4$ & 498 & $349(70.1)$ & $140(28.1)$ & $\longmapsto$ & $0.553(0.438-0.698)$ & $<0.001^{*}$ & $\longmapsto$ & $0.502(0.391-0.646)$ & $<0.001^{*}$ \\
\hline \multicolumn{10}{|l|}{$\mathrm{N}$ stage } \\
\hline No & 1040 & $819(78.8)$ & $206(19.8)$ & $\mapsto$ & $0.566(0.467-0.685)$ & $<0.001^{*}$ & $\longmapsto$ & $0.542(0.431-0.682)$ & $<0.001^{*}$ \\
\hline $\mathrm{N} 1 / \mathrm{N} 2$ & 303 & $188(62.0)$ & $111(36.6)$ & $\longmapsto$ & $0.594(0.449-0.786)$ & $<0.001^{*}$ & $\longmapsto$ & $0.550(0.415-0.730)$ & $<0.001^{*}$ \\
\hline \multicolumn{10}{|l|}{ M stage } \\
\hline MO & 1245 & $968(77.8)$ & $258(20.7)$ & $\longmapsto$ & $0.625(0.525-0.744)$ & $<0.001^{*}$ & $\longmapsto$ & $0.634(0.494-0.738)$ & $<0.001^{*}$ \\
\hline M1 & 158 & $60(38.0)$ & $96(60.8)$ & $\longmapsto$ & $0.854(0.603-1.209)$ & 0.374 & $\longmapsto$ & $0.925(0.667-1.283)$ & 0.640 \\
\hline \multicolumn{10}{|l|}{ Histology } \\
\hline $\operatorname{scc}$ & 437 & $300(68.6)$ & $130(29.7)$ & $\longmapsto$ & $0.554(0.427-0.719)$ & $<0.001^{*}$ & $\longmapsto$ & $0.545(0.406-0.732)$ & $<0.001^{*}$ \\
\hline TCC & 660 & $503(76.2)$ & $146(22.1)$ & $\mapsto$ & $0.488(0.391-0.607)$ & $<0.001^{*}$ & $\longmapsto$ & $0.530(0.409-0.686)$ & $<0.001^{*}$ \\
\hline$A C$ & 252 & $188(74.6)$ & $59(23.4)$ & $\longmapsto$ & $0.393(0.275-0.560)$ & $<0.001^{*}$ & $\longmapsto$ & $0.386(0.260-0.573)$ & $<0.001^{*}$ \\
\hline Other & 195 & $123(63.1)$ & $68(34.9)$ & $\longmapsto$ & $0.713(0.492-1.033)$ & 0.074 & $\longmapsto$ & $0.688(0.461-1.026)$ & 0.067 \\
\hline \multicolumn{10}{|c|}{ AJCC 8th stage } \\
\hline $\mid / \|$ & 660 & $546(82.7)$ & $106(16.1)$ & $\longmapsto$ & $0.708(0.538-0.931)$ & $0.013^{*}$ & $\longmapsto$ & $0.690(0.490-0.972)$ & $0.034^{*}$ \\
\hline III IV & 656 & $441(67.2)$ & $206(31.4)$ & $\mapsto$ & $0.524(0.430-0.639)$ & $<0.001^{*}$ & $\longmapsto$ & $0.507(0.411-0.626)$ & $<0.001^{*}$ \\
\hline \multicolumn{10}{|c|}{ Tumor size, mm } \\
\hline$<30$ & 230 & $196(85.2)$ & $32(13.9)$ & $\longmapsto$ & $0.604(0.350-1.041)$ & 0.070 & $\longmapsto$ & $0.412(0.224-0.724)$ & $0.002^{*}$ \\
\hline$\geq 30$ & 412 & $314(76.2)$ & $92(22.3)$ & $\longmapsto$ & $0.570(0.431-0.754)$ & $<0.001^{*}$ & $\longmapsto$ & $0.570(0.431-0.754)$ & $<0.001^{*}$ \\
\hline \multicolumn{10}{|l|}{ Sex } \\
\hline Male & 971 & $718(73.9)$ & $233(24.0)$ & $\longmapsto$ & $0.561(0.469-0.762)$ & $<0.001^{*}$ & $\longmapsto$ & $0.561(0.469-0.762)$ & $<0.001^{*}$ \\
\hline Female & 573 & $396(69.1)$ & $170(29.7)$ & $\mapsto$ & $0.464(0.370-0.581)$ & $<0.001^{*}$ & $\longmapsto$ & $0.464(0.370-0.581)$ & $<0.001^{*}$ \\
\hline
\end{tabular}

Fig. 2 Forest plots summarizing the HRs and 95\% Cls of OS and CSS in PUC patients stratified by surgical procedure 
$[2,18]$. Subset analyses in our study demonstrated that PUC patients with the predominant histological types who underwent surgery of the primary site showed a significant survival advantage, while PUC patients with rare histological types were less likely to benefit from surgery. The results underscore the continued importance of improved guidelines for management of patients with rare PUC pathological types.

Despite several promising results, this registry-based study has unavoidable limitations. First, limitations of SEER-based studies included the absence of detail with regard to individual information about chemotherapy regimen and radiotherapy doses/fields. Thus, it was not possible to examine the effects of combined surgery and chemotherapy or radiotherapy on patient survival. Second, SEER also lacked information regarding the location of PUC, a significant prognostic factor that undoubtedly influences the treatment strategy. Moreover, this study is a retrospective analysis, and selection bias could not be completely avoided.

\section{Conclusion}

Despite the limitations of this study, our results suggest that surgery for the primary tumor conferred a survival advantage in non-metastatic PUC patients regardless of sex, age, $\mathrm{T}$ stage, and $\mathrm{N}$ stage. Furthermore, the surgical benefit was more marked in patients with early TNM stage (I/II) disease, patients $<70$ years, and those with tumor size $\geq 30 \mathrm{~mm}$.

\section{Abbreviations}

PUC: Primary urethral carcinoma; OS: Overall survival; CSM: Cancer-specific mortality; CSS: Cancer-specific survival; SEER: The Surveillance, Epidemiology and End Results database; ICD-O-3: The International Classification of Diseases-O-3; AJCC: American Joint Committee on Cancer; HR: Hazard ratio; Cl: Confidence interval; Ref: Reference

\section{Supplementary Information}

The online version contains supplementary material available at https://doi. org/10.1186/s12885-021-08603-z.

Additional file 1: Supplementary Figure 1. OS and CSM in PUC patients stratified by surgical procedure and age. (a) Patients aged $<70$ years, (b) patients aged $\geq 70$ years.

Additional file 2: Supplementary Figure 2. OS and CSM in PUC patients stratified by surgical procedure and tumor size. (a) Tumor size < $30 \mathrm{~mm}$, (b) tumor size $\geq 30 \mathrm{~mm}$.

Additional file 3: Supplementary Figure 3. OS and CSM in PUC patients stratified by surgical procedure and sex. (a) Male, (b) female.

Additional file 4: Supplementary Figure 4. OS and CSM in PUC patients stratified by surgical procedure and AJCC stage groups. (a) I/II stage, (b) III/IV stage.

Additional file 5: Supplementary Figure 5. OS and CSM in PUC patients stratified by surgical procedure and M stage. (a) Stage M0, (b) stage M1.

\section{Acknowledgements}

We sincerest thank the editor and reviewers for careful review and valuable comments. The manuscript has certainly benefited from these insightful revision suggestions.

\section{Authors' contributions}

JW performed all statistical analyses and was a major contributor in manuscript writing. Y-CW conducted the data collection and was involved in the manuscript writing. $W-J L$ and $B D$ were involved in the manuscript editing. D-WY and Y-PZ conceived and designed the study. All authors read and approved the final manuscript. The funding body did not play any roles in the design of the study and collection, analysis, and interpretation of data and in writing the manuscript.

\section{Funding}

This work was supported by the National Natural Science Foundation of China (Project 81772706).

\section{Availability of data and materials}

The data that support the findings of this study are openly available in the Surveillance, Epidemiology and End Results (SEER) database of the National Cancer Institute.

\section{Declarations}

Ethics approval and consent to participate

The current study does not contain any studies with human participants or animals performed by any of the authors.

\section{Consent for publication}

Not applicable.

\section{Competing interests}

The authors declare no conflict of interest.

\section{Author details}

'Department of Urology, Fudan University Shanghai Cancer Center, No. 270 Dong an Road, Shanghai 200032, People's Republic of China. ${ }^{2}$ Department of Oncology, Shanghai Medical College, Fudan University, Shanghai, China.

Received: 17 October 2020 Accepted: 15 July 2021

Published online: 27 July 2021

References

1. Amin MB, Young RH. Primary carcinomas of the urethra. Semin Diagn Pathol. 1997;14(2):147-60.

2. Dayyani F, Hoffman K, Eifel P, Guo C, Vikram R, Pagliaro LC, et al. Management of advanced primary urethral carcinomas. BJU Int. 2014;114(1): 25-31. https://doi.org/10.1111/bju.12630.

3. Siegel RL, Miller KD, Jemal A. Cancer statistics, 2020. CA Cancer J Clin. 2020; 70(1):7-30. https://doi.org/10.3322/caac.21590.

4. Grigsby PW. Carcinoma of the urethra in women. Int J Radiat Oncol Biol Phys. 1998:41(3):535-41. https://doi.org/10.1016/S0360-3016(97)00773-6.

5. Dalbagni G, Zhang ZF, Lacombe L, Herr HW. Male urethral carcinoma: analysis of treatment outcome. Urology. 1999;53(6):1126-32. https://doi. org/10.1016/S0090-4295(98)00659-1.

6. Sui W, RoyChoudhury A, Wenske S, Decastro GJ, McKiernan JM, Anderson CB. Outcomes and prognostic factors of primary urethral Cancer. Urology. 2017;100:180-6. https://doi.org/10.1016/j.urology.2016.09.042.

7. Gakis G, Bruins HM, Cathomas R, Comperat EM, Cowan NC, van der Heijden $A G$, et al. European Association of Urology guidelines on primary urethral Carcinoma-2020 update. Eur Urol Oncol. 2020;3(4):424-32.

8. Dimarco DS, Dimarco CS, Zincke H, Webb MJ, Bass SE, Slezak JM, et al. Surgical treatment for local control of female urethral carcinoma. Urol Oncol. 2004;22(5):404-9. https://doi.org/10.1016/S1078-1439(03)00174-1.

9. Cohen MS, Triaca V, Billmeyer B, Hanley RS, Girshovich L, Shuster T, et al. Coordinated chemoradiation therapy with genital preservation for the treatment of primary invasive carcinoma of the male urethra. J Urol. 2008; 179(2):536-41; discussion 41. https://doi.org/10.1016/j.juro.2007.09.068.

10. Wei Y, Wu YP, Xu N, Li XD, Chen SH, Cai H, et al. Sex-related differences in clinicopathological features and survival of patients with primary urethral carcinoma: a population-based study. Onco Targets Ther. 2017;10:3381-9. https://doi.org/10.2147/OTT.S139252.

11. Scrucca L, Santucci A, Aversa F. Competing risk analysis using R: an easy guide for clinicians. Bone Marrow Transplant. 2007:40(4):381-7. https://doi. org/10.1038/sj.bmt.1705727. 
12. Kuk D, Varadhan R. Model selection in competing risks regression. Stat Med. 2013;32(18):3077-88. https://doi.org/10.1002/sim.5762.

13. Swartz MA, Porter MP, Lin DW, Weiss NS. Incidence of primary urethral carcinoma in the United States. Urology. 2006;68(6):1164-8. https://doi.org/1 0.1016/j.urology.2006.08.1057.

14. Dayyani F, Pettaway CA, Kamat AM, Munsell MF, Sircar K, Pagliaro LC. Retrospective analysis of survival outcomes and the role of cisplatin-based chemotherapy in patients with urethral carcinomas referred to medical oncologists. Urol Oncol. 2013;31(7):1171-7. https://doi.org/10.1016/j. urolonc.2012.01.011.

15. Gakis G, Schubert T, Morgan TM, Daneshmand S, Keegan KA, Mischinger J, et al. The prognostic effect of salvage surgery and radiotherapy in patients with recurrent primary urethral carcinoma. Urol Oncol. 2018;36(1):10 e7-10 e14.

16. Derksen JW, Visser O, de la Riviere GB, Meuleman EJ, Heldeweg EA, Lagerveld BW. Primary urethral carcinoma in females: an epidemiologic study on demographical factors, histological types, tumour stage and survival. World J Urol. 2013;31(1):147-53. https://doi.org/10.1007/s00345-0120882-5.

17. Gakis G, Morgan TM, Efstathiou JA, Keegan KA, Mischinger J, Todenhoefer T, et al. Prognostic factors and outcomes in primary urethral cancer: results from the international collaboration on primary urethral carcinoma. World J Urol. 2016;34(1):97-103. https://doi.org/10.1007/s00345-015-1583-7.

18. Abudurexiti M, Wang J, Shao N, Wan FN, Zhu Y, Dai B, et al. Prognosis of rare pathological primary urethral carcinoma. Cancer Manag Res. 2018;10: 6815-22. https://doi.org/10.2147/CMAR.S184197.

\section{Publisher's Note}

Springer Nature remains neutral with regard to jurisdictional claims in published maps and institutional affiliations.

Ready to submit your research? Choose BMC and benefit from:

- fast, convenient online submission

- thorough peer review by experienced researchers in your field

- rapid publication on acceptance

- support for research data, including large and complex data types

- gold Open Access which fosters wider collaboration and increased citations

- maximum visibility for your research: over $100 \mathrm{M}$ website views per year

At $\mathrm{BMC}$, research is always in progress.

Learn more biomedcentral.com/submissions 